\title{
Serum Angiogenesis Markers and Their Correlation with Ultrasound-Detected Synovitis in Juvenile Idiopathic Arthritis
}

\author{
Joanna Świdrowska, ${ }^{1}$ Piotr Smolewski, ${ }^{2}$ Jerzy Stańczyk, ${ }^{1}$ and Elżbieta Smolewska ${ }^{1}$ \\ ${ }^{1}$ Department of Pediatric Cardiology and Rheumatology, Medical University of Lodz, 91-738 Lodz, Poland \\ ${ }^{2}$ Department of Experimental Hematology, Medical University of Lodz, 93-513 Lodz, Poland \\ Correspondence should be addressed to Elżbieta Smolewska; e.smolewska@wp.pl
}

Received 8 February 2015; Accepted 13 April 2015

Academic Editor: David Kaplan

Copyright (c) 2015 Joanna Świdrowska et al. This is an open access article distributed under the Creative Commons Attribution License, which permits unrestricted use, distribution, and reproduction in any medium, provided the original work is properly cited.

\begin{abstract}
Synovial angiogenesis is considered to be an important early step in the pathogenesis of juvenile idiopathic arthritis (JIA). In this study we assessed levels of angiogenic markers in serum or synovial fluid and their possible relevance to disease activity or degree of ultrasound signs of synovial inflammation and angiogenesis in early JIA. The concentration of vascular endothelial growth factor (VEGF), its soluble receptors 1 and 2 (sVEGF-R1, sVEGF-R2), and angiopoietins 1 and 2 (ANG-1, ANG-2) were evaluated in 43 JIA patients and 23 healthy controls. Synovial angiogenesis was assessed by means of Power-Doppler Ultrasonography (PDUS), according to the fourth-grade vascularity scale. VEGF and its receptors' (sVEGF-R1, sVEGF-R2) serum levels were significantly higher in JIA patients $(p=0.002)$. We found large variation in serum ANG-1 and ANG-2 levels. The PDUS imaging identified increased synovial microvascular blood flow in 15 (35.7\%) examined JIA children. Intensity of joint vascularization correlated with higher serum VEGF and its levels was lowest in grade 0 and highest in grade 3 ( $p<0.007$ and $p<0.001$, resp.). In conclusion, the high correlation between synovial microvascular blood flow, serum angiogenic proteins, and symptoms of synovitis may indicate its important role in pathogenesis of JIA.
\end{abstract}

\section{Introduction}

Juvenile idiopathic arthritis (JIA) is the most common childhood chronic rheumatic disease, characterized by arthritis of unknown origin with onset before the age of 16 years $[1,2]$. The inflammatory process in JIA is characterized by excessive proliferation of synoviocytes. It is strongly associated with a neovascularization due to increased metabolic requirement of hypertrophic synovium [3]. Neovascularization, also defined as angiogenesis, is a complex process in which new capillaries develop from preexisting vasculature due to hypoxemia, injury, or inflammation of the tissues. This process plays a pivotal role in a number of physiological and pathological conditions, including chronic inflammatory diseases [4]. Angiogenesis is regulated by the imbalance between proangiogenic and antiangiogenic factors [5] and occurs in the first stage of synovitis. Vascular endothelial growth factor (VEGF) is the best known and the most endothelial cellspecific angiogenic factor [6]. The VEGF family currently consists of six members: VEGF-A, VEGF-B, VEGF-C, VEGF$D$, VEGF-E, and placenta growth factor. Soluble VEGF receptor-1 (sVEGF-R1; sFlt-1) regulates the biologic activity of VEGF and it is one of the three specific receptors that mediate the actions of VEGF [7]. Angiopoietins (ANGs), less explored in the pathogenesis of chronic inflammatory connective tissue diseases, are known as the mediators of angiogenesis regulating endothelial integrity and inflammation.

In adult rheumatoid arthritis (RA) VEGF has been described as a crucial factor of neovascularisation at the stage of hypertrophic synovium [8]. It has been found in higher concentrations in serum and synovial fluid and there was a correlation found between its levels and disease activity [9]. Now, there have been very few studies published investigating the production of VEGF in the inflamed joints of children with JIA.

Recently, visualization of hypertrophic synovium and its vasculature has become possible by high resolution ultrasound (US) imaging and the Power-Doppler (PD) 
mode (PDUS). Technical improvements of ultrasonography allowed detecting the low-velocity blood flow in synovial microvessels. High sensitivity of synovitis US in RA has been already shown in several publications considering adult rheumatology $[10,11]$. Unfortunately, there are only few reports discussing that issue in pediatric rheumatology [12].

The purpose of the study was to discuss the role of serum vascular angiogenic factors: VEGF, its soluble receptors, and ANGs 1 and 2 (ANG-1, ANG-2, resp.) in pathophysiology of JIA. Importantly, we also aimed to investigate the intraarticular synovial microvascular blood flow in clinically symptomatic JIA joints by means of PDUS in correlation with biological markers of inflammation and angiogenic factors.

\section{Materials and Methods}

2.1. Patients. Forty-three children who met the International League of Associations for Rheumatology (ILAR) criteria for JIA [13], 31 girls and 12 boys, median age 9 years (range 1.5-17 years), were included into the study. All had an early diagnosis of JIA determined by a consultant pediatric rheumatologist. All children were without any oral/iv corticosteroid history. None of children had received any intra-articular steroid injection in the past. The control group consisted of 23 healthy children, 15 girls and 8 boys, median age 10.5 (range 417.5). The study was approved by the local Ethics Committee.

2.2. Clinical and Laboratory Assessment. Each child had a detailed clinical history taken. The following data were recorded for each patient at the study visit: sex, age, ILAR category, disease duration, current treatment, and active joints count. A joint with active disease was defined as the presence of swelling or tenderness/pain on motion and restricted motion [14]. Activity of JIA was established based on the 27-joint Juvenile Arthritis Disease Activity Score (JADAS27) [15]. Low, intermediate, and high stages of the disease activity have been distinguished accordingly. In the study group 27 (62.8\%) children had low, 10 (23.25\%) medium, and $6(13.95 \%)$ high activity of the disease (Table 1). All the examined patients had a normal renal function. Majority of the study group comprised children with oligoarthritis type of JIA onset (30 patients, $69.8 \%$ ), whereas 13 children $(30.2 \%$ ) represented polyarticular and only $3(6 \%)$ systemic disease. The type of onset was defined according to ILAR criteria (2001) [16].

Clinical evaluation was performed by two pediatric rheumatologists prior to laboratory testing or ultrasound examination. Laboratory assessment included determination of erythrocyte sedimentation ratio (ESR) by Westergren method and C-reactive protein (CRP) level using the immunoturbidimetric method. Normal values for these laboratory methods were for ESR $<12 \mathrm{~mm} / \mathrm{h}$ and for CRP $0-5.0 \mathrm{mg} / \mathrm{dL}$. Results of antinuclear antibodies (ANA) and rheumatoid factor (RF) were also recorded, using standard methods.
TABLE 1: Characteristics of the examined group.

\begin{tabular}{lc}
\hline JIA children & $N(\%) / \mathrm{Me}$ (range) \\
\hline Total number & 43 \\
Sex & $31(72.1 \%)$ \\
$\quad$ Girls & $12(27.9 \%)$ \\
$\quad$ Boys & $9(1.5-17)$ years \\
Age & $7(0.5-120)$ months \\
Time of JIA duration & \\
Type of onset & $30(69.8 \%)$ \\
$\quad$ Oligoarthritis & $13(30.2 \%)$ \\
Polyarthritis & $27(62.8 \%)$ \\
Disease activity & $10(23.25 \%)$ \\
Low & $6(13.95 \%)$ \\
Moderate & $7.7(3.0-19.0)$ G/L \\
High & $355.0(156.0-613.0)$ G/L \\
WBC & $21(2-130)$ after $1 \mathrm{~h}$ \\
PLT & $2.58(0.05-148.6)$ \\
ESR & $19(44.2 \%)$ \\
CRP & $28(65.1 \%)$ \\
ANA > 1:160 & Types 1-26, types 2-11, types 3-6 \\
RF $>14$ IU & $3-15(34.9 \%)$ \\
US of joints* & Grades $0-6(13.95 \%)$, grades $1-8$ \\
RTG of hands & grades $2-14(32.55 \%)$, grades \\
\hline
\end{tabular}

${ }^{*}$ Joint vascularization scale: grade $0=$ no signs of vascularization, $1=$ mild (presence of single/vessel dots), 2 = moderate (presence of confluent vessel dots in less than half of the synovial area), and $3=$ marked (presence of confluent vessel dots in more than half of the synovial area).

2.3. VEGF, sVEGF-R1, sVEGF-R2, ANG-1, and ANG-2 Levels. Levels of human VEGF, human angiopoietins (ANG-1, ANG2), and human soluble VEGF receptors 1 and 2 (sVEGF-R1 and sVEGF-R2, resp.) were measured by ELISA in serum samples from JIA patients and 23 age-matched healthy controls. Moreover, in 8 patients concentrations of these proteins in synovial fluid were measured. VEGF, sVEGF-R1, sVEGFR2, ANG-1, and ANG-2 were assessed using a standard quantitative sandwich ELISA (Quantikine, R\&D Systems, Lille, France) according to manufacturer's instructions.

2.4. Ultrasonography (US). US assessment was performed in the same hospitalization with clinical and laboratory examination, by a clinician experienced in musculoskeletal ultrasonography. A Philips CX50 CompactXtreme ultrasound system and a $5-12 \mathrm{MHz}$ linear transducer were used in this study. The examination was performed in a minimum of two planes (longitudinal and transverse) and included the following joints: knees, ankles, wrists, elbows, metacarpophalangeal joints (MCP), proximal interphalangeal joints (PIP), metatarsophalangeal joints (MTP), and midfoot. The diagnosis of US-detected synovitis using gray-scale US was defined by the presence of synovial hypertrophy and/or the presence of a compressible anechoic space within the joint, 


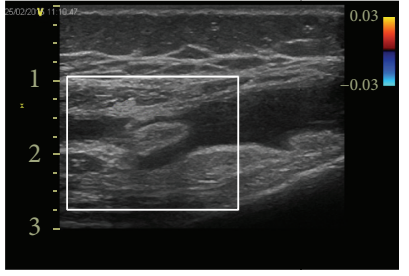

Grade 0

(a)

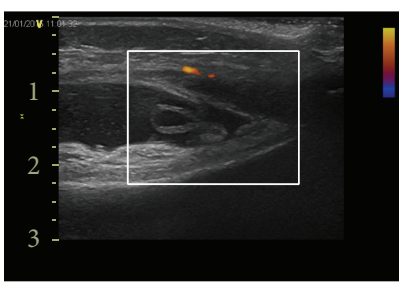

Grade 1

(b)

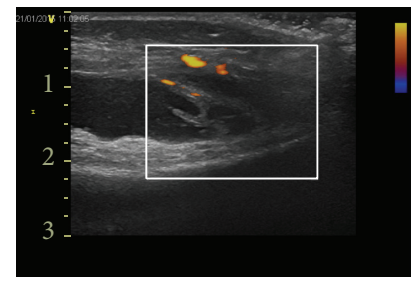

Grade 2

(c)

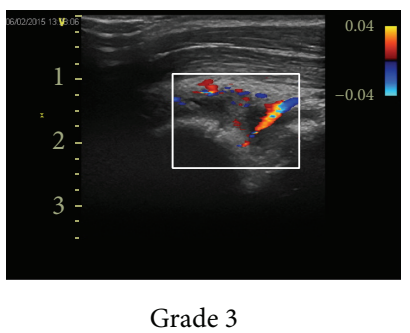

(d)

FIGURE 1: Ultrasound evaluation of joint vascularization scale using Power-Doppler Ultrasonography imaging. Examples of visualization of different degrees of joints inflammation and angiogenesis. Joint vascularization scale: grade $0=$ no signs of vascularization, $1=$ mild (presence of single/vessel dots), 2 = moderate (presence of confluent vessel dots in less than half of the synovial area), and $3=$ marked (presence of confluent vessel dots in more than half of the synovial area).

representing fluid [17]. Synovitis detected by US was graded as mild, moderate, or severe (score from 0 to 3 ). Hypertrophic synovium was scanned for the presence of Power-Doppler signal, standardized with a lower pulse repetition frequency of $750 \mathrm{MHz}$ and low wall filters. The color gain was increased to the highest values not generating PD signals under the bony cortex [18]. PDUS signal was scored on semiquantitative four-grade scale: $0=$ no signs of vascularization, $1=$ mild (presence of single/vessel dots), $2=$ moderate (presence of confluent vessel dots in less than half of the synovial area), and $3=$ marked (presence of confluent vessel dots in more than half of the synovial area) (Figure 1).

2.5. Statistics. For the statistical analysis of data obtained, the range of the measured variable, mean, median, and standard deviation (SD) were calculated, using statistical software (STATISTICA v.7.0, Tulsa, OK, USA). The data are presented as mean $\pm \mathrm{SD}$ values. The differences between values were evaluated with nonparametric Mann-Whitney test, where the distribution of data was normal. The correlation between features was evaluated using the Spearman rank coefficient $\rho$. The $p$ values less than 0.05 were considered statistically significant.

\section{Results}

The characteristics of the examined group are shown in Table 1.

VEGF serum levels were significantly higher in JIA patients than in healthy controls $(p=0.002$, Table 2$)$. Similar pattern was observed for sVEGF-R1 and sVEGF-R2 ( $p=$ 0.004 and $p=0.001$, resp.). There was large variation in serum ANG-1 and -2 levels in JIA patients and healthy controls, without statistically significant differences (Table 2).

In JIA children serum VEGF levels correlated significantly with CRP and ESR ( $R=0.39$ and $R=0.41$, resp.; $p<0.05)$. Serum levels of ANG-1 correlated with white blood cells (WBC) and platelets (PLT) counts ( $R=0.31$ and $R=0.55$, resp.; $p<0.05$ ), whereas ANG-2 correlated with WBC count $(R=0.32$ and $p<0.05)$. There was no correlation of neither sVEGF-R1 nor sVEGF-R2 levels and
TABLE 2: Serum levels of angiogenic proteins in JIA in comparison to healthy children.

\begin{tabular}{lccc}
\hline Parameter & Ctrl & JIA & $p$ \\
& $N=23$ & $N=43$ & \\
\hline VEGF & $0.21 \pm 0.16$ & $0.64 \pm 0.63$ & $\mathbf{0 . 0 0 2}$ \\
R1 & $0.09 \pm 0.03$ & $0.11 \pm 0.03$ & $\mathbf{0 . 0 0 4}$ \\
R2 & $10.34 \pm 4.47$ & $15.92 \pm 7.10$ & $\mathbf{0 . 0 0 1}$ \\
ANG-1 & $48.85 \pm 16.39$ & $50.01 \pm 17.10$ & NS \\
ANG-2 & $1.81 \pm 1.44$ & $6.34 \pm 34.12$ & NS \\
\hline
\end{tabular}

TABLE 3: Correlation between angiogenic proteins and laboratory parameters in JIA children.

\begin{tabular}{lccccc}
\hline Variable & VEGF & R1 & R2 & ANG-1 & ANG-2 \\
\hline WBC & 0.13 & 0.21 & 0.16 & $\mathbf{0 . 3 1}$ & $\mathbf{0 . 3 2}$ \\
PLT & 0.22 & 0.18 & 0.28 & $\mathbf{0 . 5 5}$ & 0.10 \\
CRP & $\mathbf{0 . 3 9}$ & -0.07 & -0.06 & 0.26 & 0.26 \\
ESR & $\mathbf{0 . 4 1}$ & 0.07 & 0.07 & 0.17 & 0.13 \\
\hline
\end{tabular}

examined laboratory parameters (Table 3 ). There were no statistically significant differences in examined markers of angiogenesis and disease activity, type of JIA onset, and the disease duration.

Comparison between concentrations of angiogenic proteins in serum and synovial fluid showed significantly higher levels of VEGF and sVEGF-R1 in synovial fluid $(p=0.004$ and $p<0.001$, resp.), whereas sVEGF-R2 and ANG-1 were higher in serum of JIA children $(p=0.008$ and $p<0.001$, resp.). ANG-2 concentration in serum and synovial fluid was similar (Table 4).

The PD mode identified marked synovial microvascular blood flow in joints (grade 3 vascularization) in 15 JIA patients (35.7\%). There were no significant differences in angiogenic protein levels between grades 0 and 1 and grades 2 and 3 . VEGF levels were lowest in grades $0 / 1$ and highest in grade 3 $(p<0.007$; Figure 2$)$. ANG-2 levels were significantly higher in grade 3 than in 0,1 , or $2(p<0.001)$. Interestingly, sVEGFR1 levels in grade 2 were statistically lower than in grade 0 $(p=0.030)$. 


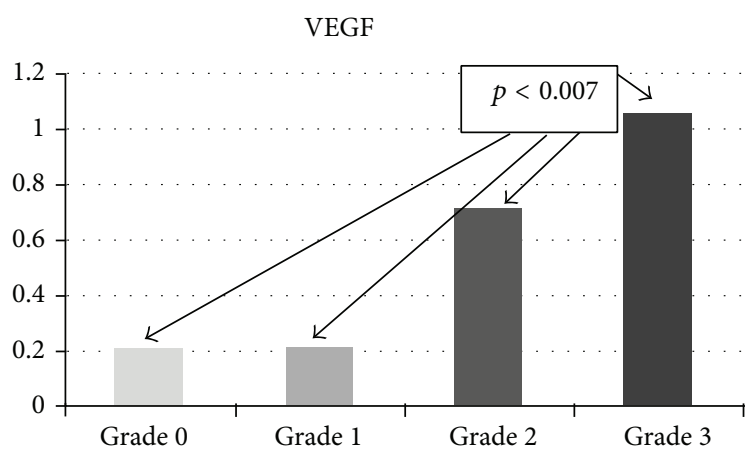

sVEGF-R2

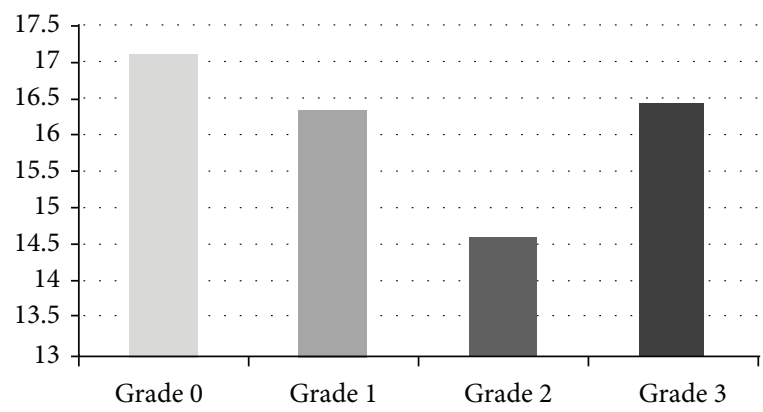

ANG-1

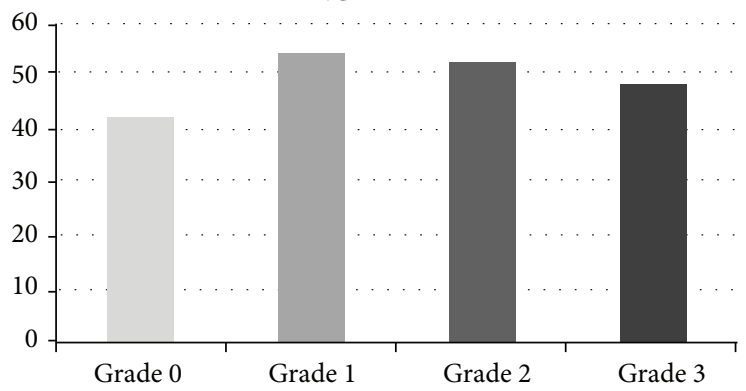

sVEGF-R1

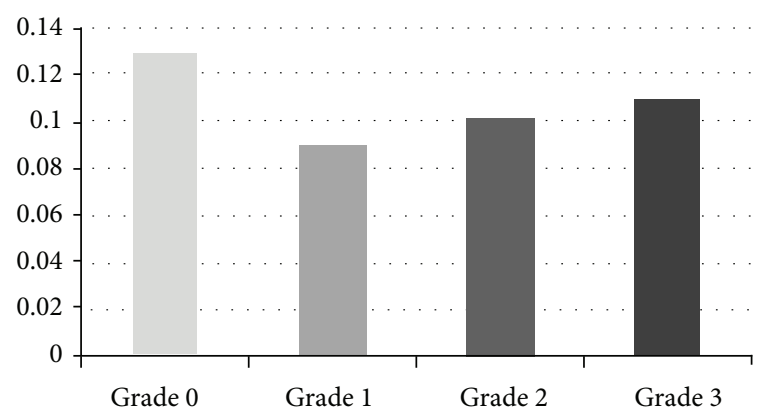

ANG-2

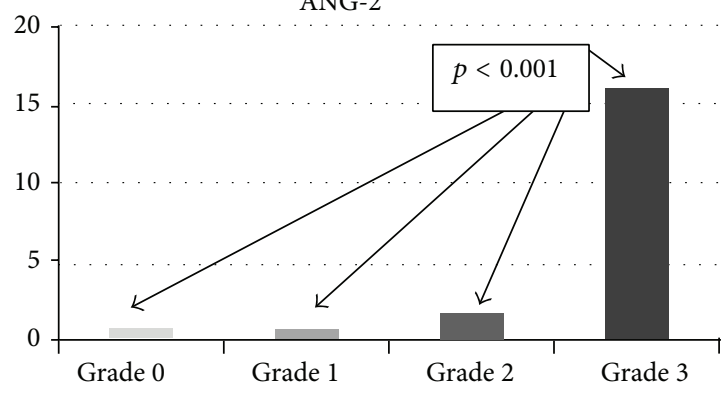

FIGURE 2: Ultrasound joint vascularization scale and serum levels of angiogenic proteins in JIA children. Joint vascularization scale: grade $0=$ no signs of vascularization, $1=$ mild (presence of single/vessel dots), $2=$ moderate (presence of confluent vessel dots in less than half of the synovial area), and $3=$ marked (presence of confluent vessel dots in more than half of the synovial area).

There were no statistically significant differences between angiogenic protein levels and radiological picture of joints in JIA children.

\section{Discussion}

Angiogenesis starts very early in rheumatoid process and is controlled by a variety of stimulators and inhibitors [19]. It is crucial to chronic inflammation and implies being essential pathogenetic mechanism in both the establishment and persistence of RA and, probably, JIA [20]. In this study we assessed five angiogenesis markers in patients with JIA. Among them were ANG-1 and ANG-2, to the best of our knowledge for the first time evaluated in JIA. Levels of these markers were correlated with signs of synovial inflammation measured using PDUS imaging.
Elevated serum levels of VEGF and its receptors have been previously shown in adult patients with RA [21, 22]. Among the angiogenesis stimulators, we found significantly higher serum VEGF levels and its soluble receptors (sVEGF-R1 and sVEGF-R2) in JIA children than in healthy controls. These results are in agreement with two other studies investigating VEGF in this disease [12, 23]. Moreover, Lee et al. [24] showed no correlation between VEGF levels in serum and synovial fluid from the same RA patient. Our study revealed the higher concentration of VEGF and sVEGF-R1 and, inversely, lower concentration of sVEGF-R2 and ANG-1 synovial fluid of JIA patients.

The other angiogenesis stimulators investigated in our study were ANG-1 and ANG-2. The role of ANG-1 is the stabilization of new capillaries, while the role of ANG-2 is antagonistic. Namely, ANG-2 blocks the vessel maturation 
TABLE 4: Comparison between angiogenic protein levels in peripheral serum and synovial fluid.

\begin{tabular}{lccc}
\hline Parameter & Serum & $\begin{array}{c}\text { Synovial fluid } \\
N=8\end{array}$ & $p$ \\
\hline VEGF & $N=43$ & $1.84 \pm 1.54$ & $\mathbf{0 . 0 0 4}$ \\
sVEGF-R1 & $0.64 \pm 0.63$ & $0.46 \pm 0.55$ & $<\mathbf{0 . 0 0 1}$ \\
sVEGF-R2 & $15.92 \pm 7.10$ & $8.70 \pm 4.82$ & $\mathbf{0 . 0 0 8}$ \\
ANG-1 & $50.01 \pm 17.10$ & $5.78 \pm 3.68$ & $<\mathbf{0 . 0 0 1}$ \\
ANG-2 & $6.34 \pm 34.12$ & $6.06 \pm 7.81$ & NS \\
\hline
\end{tabular}

[25]. As was mentioned, there are no studies considering the role of ANGs in JIA. Available data regarding ANGs' expression in adult patients with RA are unclear [26, 27]. We did not find significant differences in ANGs' serum levels between JIA and healthy children.

Besides the increase of some angiogenesis stimulators serum levels in JIA patients, our study showed also their correlation with biomarkers of inflammation. Interestingly, VEGF level was strongly correlated with CRP and ESR levels. In contrast, $\mathrm{ANGs}$ ' levels corresponded with WBC and PLT count. Lee et al. [24] showed the similar pattern of VEGF and interaction of inflammation markers in RA patients.

Since US imaging and PDUS have clearly exposed the inflammation and blood flow in synovium, we analyzed whether there was a correlation between angiogenic markers and the US and PDUS findings. The PDUS identified marked synovial microvascular blood flow in joints (grade 3 vascularization) in more than one-third of JIA patients. Our study has also showed the significant relationship between VEGF levels and synovial vasculature blood flow. The lowest serum levels of VEGF were found in vascularity grades $0 / 1$ and highest in grade 3 . ANG-2 serum levels were also significantly higher in grade 3. Strunk et al. [28] and Gok et al. [29] in their studies on RA patients did not find any significant correlation between serum VEGF levels and the degree of synovial vascularity. In the same study, Gok et al. [29] found the positive correlation between ANG-1 levels with effusion, but not synovial and PDUS scores. To our knowledge, there are no other studies considering the correlation between PDUS vascularity grade scale and other angiogenic factors, such as VEGF receptors and ANG-2.

In conclusion, our study strongly suggests the crucial role of angiogenesis in JIA. Serum angiogenesis modulators are associated not only with pathogenesis of JIA, but also with its severity and progression. VEGF, as the most specific angiogenesis marker, reflects the degree of inflammation in JIA patients. In compilation with VEGF level, PDUS provides early information about the synovitis activity during the course of the inflammatory process. Although the lack of specificity makes the angiogenic modulators still not applicable for diagnostic principles, their close correlation with disease severity might be essential in JIA patients monitoring and follow-up.

\section{Conflict of Interests}

The authors declare that there is no conflict of interests regarding the publication of this paper.

\section{Acknowledgment}

This work was supported in part by the grants from the Medical University of Lodz, Poland: no. 503/8-093-01/503-01 and no. 502-03/8-000/01/502-64-063.

\section{References}

[1] B. Prakken, S. Albani, and A. Martini, "Juvenile idiopathic arthritis," The Lancet, vol. 377, no. 9783, pp. 2138-2149, 2011.

[2] A. Ravelli and A. Martini, "Juvenile idiopathic arthritis," The Lancet, vol. 369, no. 9563, pp. 767-778, 2007.

[3] L. Schrieber and J. C. Jackson, "Angiogenesis in rheumatoid arthritis," in Rheumatology, H. J. Klippel and P. A. Dieppe, Eds., vol. 1, pp. 1-12, Mosby, London, UK, 2nd edition, 1988.

[4] N. Ferrara and K. Alitalo, "Clinical applications of angiogenic growth factors and their inhibitors," Nature Medicine, vol. 5, no. 12, pp. 1359-1364, 1999.

[5] P. C. Taylor and B. Sivakumar, "Hypoxia and angiogenesis in rheumatoid arthritis," Current Opinion in Rheumatology, vol. 17, no. 3, pp. 293-298, 2005.

[6] M. Clauss, "Molecular biology of the VEGF and the VEGF receptor family," Seminars in Thrombosis and Hemostasis, vol. 26, no. 5, pp. 561-569, 2000.

[7] M. J. Cross and L. Claesson-Welsh, "FGF and VEGF function in angiogenesis: signalling pathways, biological responses and therapeutic inhibition," Trends in Pharmacological Sciences, vol. 22, no. 4, pp. 201-207, 2001.

[8] E. M. Paleolog, "Angiogenesis: a critical process in the pathogenesis of RA - a role for VEGF?" British Journal of Rheumatology, vol. 35, no. 10, pp. 917-919, 1996.

[9] M. Harada, K. Mitsuyama, H. Yoshida et al., "Vascular endothelial growth factor in patients with rheumatoid arthritis," Scandinavian Journal of Rheumatology, vol. 27, no. 5, pp. 377-380, 1998.

[10] K. Kikuchi, M. Kubo, T. Kadono, N. Yazawa, H. Ihn, and K. Tamaki, "Serum concentrations of vascular endothelial growth factor in collagen diseases," British Journal of Dermatology, vol. 139, no. 6, pp. 1049-1051, 1998.

[11] A. E. Koch, L. A. Harlow, G. K. Haines et al., "Vascular endothelial growth factor: a cytokine modulating endothelial function in rheumatoid arthritis," Journal of Immunology, vol. 152, no. 8, pp. 4149-4156, 1994.

[12] S. Vignola, P. Picco, F. Falcini, F. Sabatini, A. Buoncompagni, and M. Gattorno, "Serum and synovial fluid concentration of vascular endothelial growth factor in juvenile idiopathic arthritides," Rheumatology, vol. 41, no. 6, pp. 691-696, 2002.

[13] R. E. Petty, T. R. Southwood, P. Manners et al., "International League of Associations for Rheumatology classification of juvenile idiopathic arthritis: second revision, Edmonton, 2001," Journal of Rheumatology, vol. 31, no. 2, pp. 390-392, 2004.

[14] A. Ravelli, S. Viola, N. Ruperto, B. Corsi, G. Ballardini, and A. Martini, "Correlation between conventional disease activity measures in juvenile chronic arthritis," Annals of the Rheumatic Diseases, vol. 56, no. 3, pp. 197-200, 1997.

[15] A. Consolaro, N. Ruperto, A. Bazso et al., "Development and validation of a composite disease activity score for juvenile idiopathic arthritis," Arthritis Care \& Research, vol. 61, no. 5, pp. 658-666, 2009.

[16] R. E. Petty, T. R. Southwood, P. Manners et al., "International League of Associations for Rheumatology Classification of 
Juvenile Idiopathic Arthritis: second revision, Edmonton, 2001," Journal of Rheumatology, vol. 31, no. 2, pp. 390-392, 2004.

[17] R. J. Wakefield, P. V. Balint, M. Szkudlarek et al., "Musculoskeletal ultrasound including definitions for ultrasonographic pathology," Journal of Rheumatology, vol. 32, no. 12, pp. 24852487, 2005.

[18] S. T. Torp-Pedersen and L. Terslev, "Settings and artefacts relevant in colour/power Doppler ultrasound in rheumatology," Annals of the Rheumatic Diseases, vol. 67, no. 2, pp. 143-149, 2008.

[19] Z. Szekanecz and A. E. Koch, "Mechanisms of disease: angiogenesis in inflammatory diseases," Nature Clinical Practice Rheumatology, vol. 3, no. 11, pp. 635-643, 2007.

[20] Z. Szekanecz, T. Besenyei, Á. Szentpétery, and A. E. Koch, "Angiogenesis and vasculogenesis in rheumatoid arthritis," Current Opinion in Rheumatology, vol. 22, no. 3, pp. 299-306, 2010.

[21] G. Clavel, N. Bessis, D. Lemeiter et al., "Angiogenesis markers (VEGF, soluble receptor of VEGF and angiopoietin-1) in very early arthritis and their association with inflammation and joint destruction," Clinical Immunology, vol. 124, no. 2, pp. 158-164, 2007.

[22] P. C. Taylor, "Serum vascular markers and vascular imaging in assessment of rheumatoid arthritis disease activity and response to therapy," Rheumatology, vol. 44, no. 6, pp. 721-728, 2005.

[23] N. Maeno, S. Takei, H. Imanaka et al., "Increased circulating vascular endothelial growth factor is correlated with disease activity in polyarticular juvenile rheumatoid arthritis," Journal of Rheumatology, vol. 26, no. 10, pp. 2244-2248, 1999.

[24] S.-S. Lee, Y.-S. Joo, W.-U. Kim et al., "Vascular endothelial growth factor levels in the serum and synovial fluid of patients with rheumatoid arthritis," Clinical and Experimental Rheumatology, vol. 19, no. 3, pp. 321-324, 2001.

[25] Z. Szekanecz and A. E. Koch, "Angiogenesis and its targeting in rheumatoid arthritis," Vascular Pharmacology, vol. 51, no. 1, pp. $1-7,2009$.

[26] U. Fearon, K. Griosios, A. Fraser et al., "Angiopoietins, growth factors, and vascular morphology in early arthritis," The Journal of Rheumatology, vol. 30, no. 2, pp. 260-268, 2003.

[27] S. Shahrara, M. V. Volin, M. A. Connors, G. K. Haines, and A. E. Koch, "Differential expression of the angiogenic Tie receptor family in arthritic and normal synovial tissue," Arthritis Research, vol. 4, no. 3, pp. 201-208, 2002.

[28] J. Strunk, E. Heinemann, G. Neeck, K. L. Schmidt, and U. Lange, "A new approach to studying angiogenesis in rheumatoid arthritis by means of Power Doppler ultrasonography and measurement of serum vascular endothelial growth factor," Rheumatology, vol. 43, no. 12, pp. 1480-1483, 2004.

[29] M. Gok, H. Erdem, F. Gogus et al., "Relationship of ultrasonographic findings with synovial angiogenesis modulators in different forms of knee arthritides," Rheumatology International, vol. 33, no. 4, pp. 879-885, 2013. 


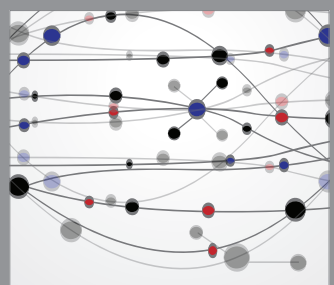

The Scientific World Journal
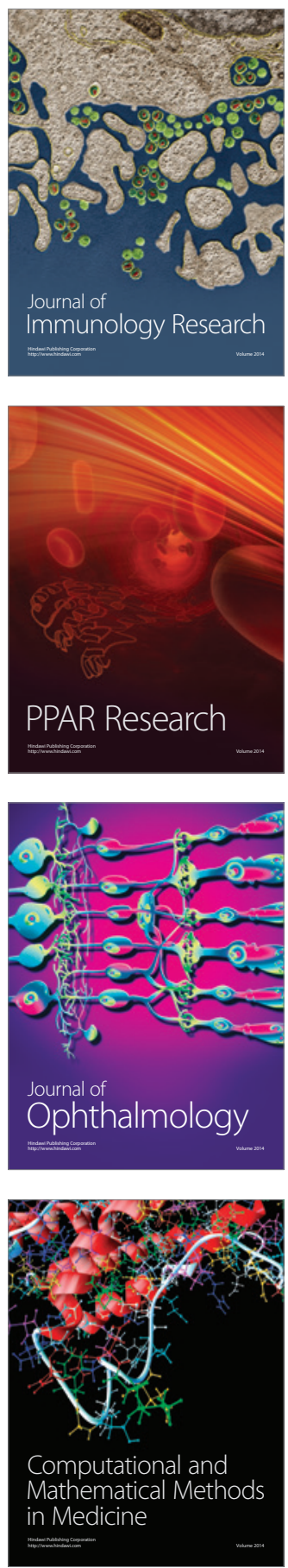

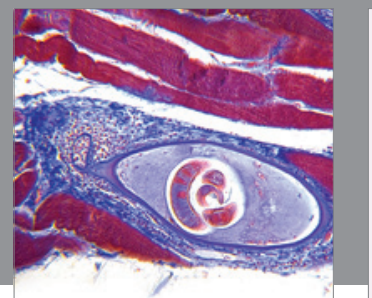

Gastroenterology

Research and Practice
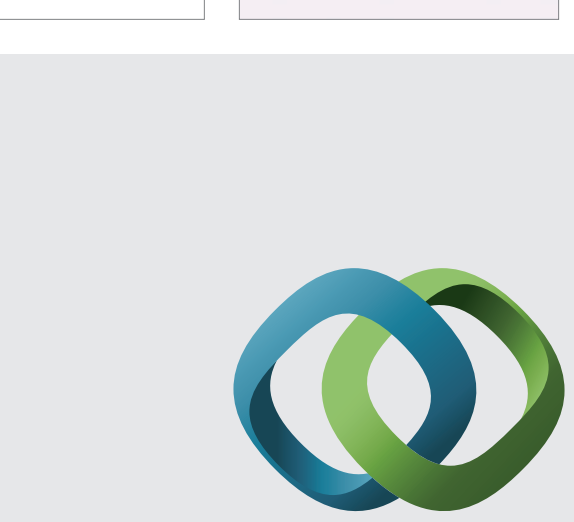

\section{Hindawi}

Submit your manuscripts at

http://www.hindawi.com
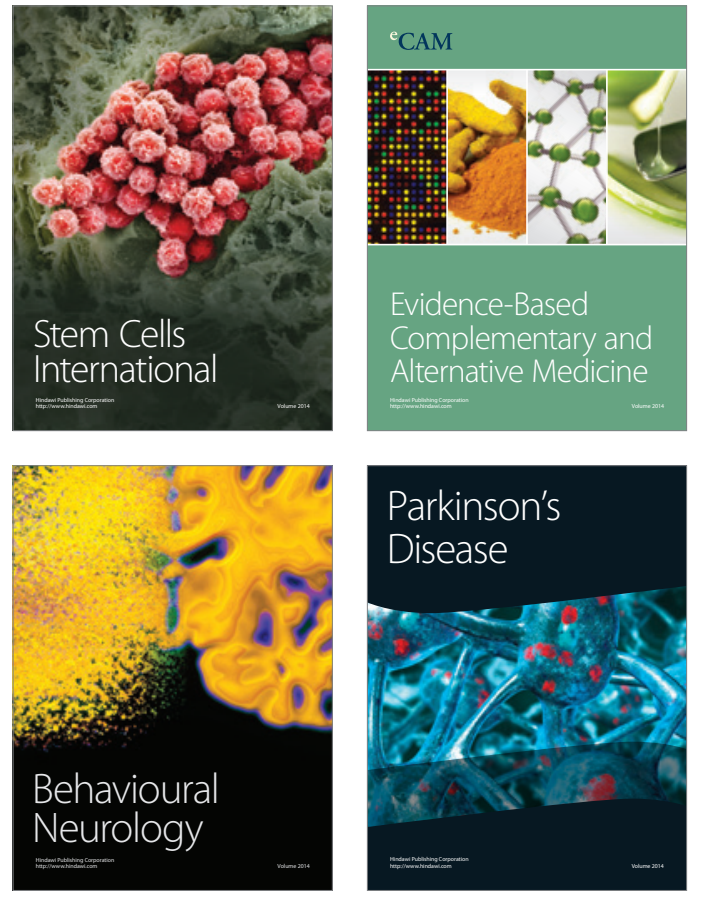
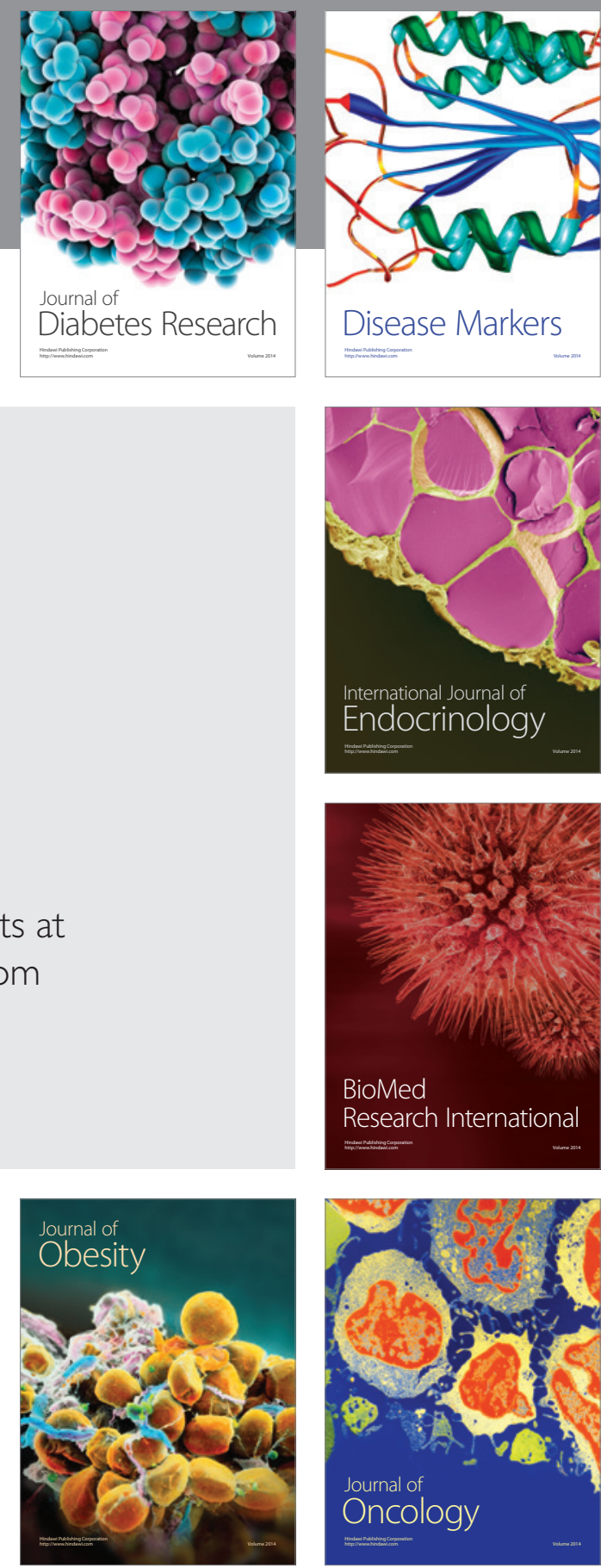

Disease Markers
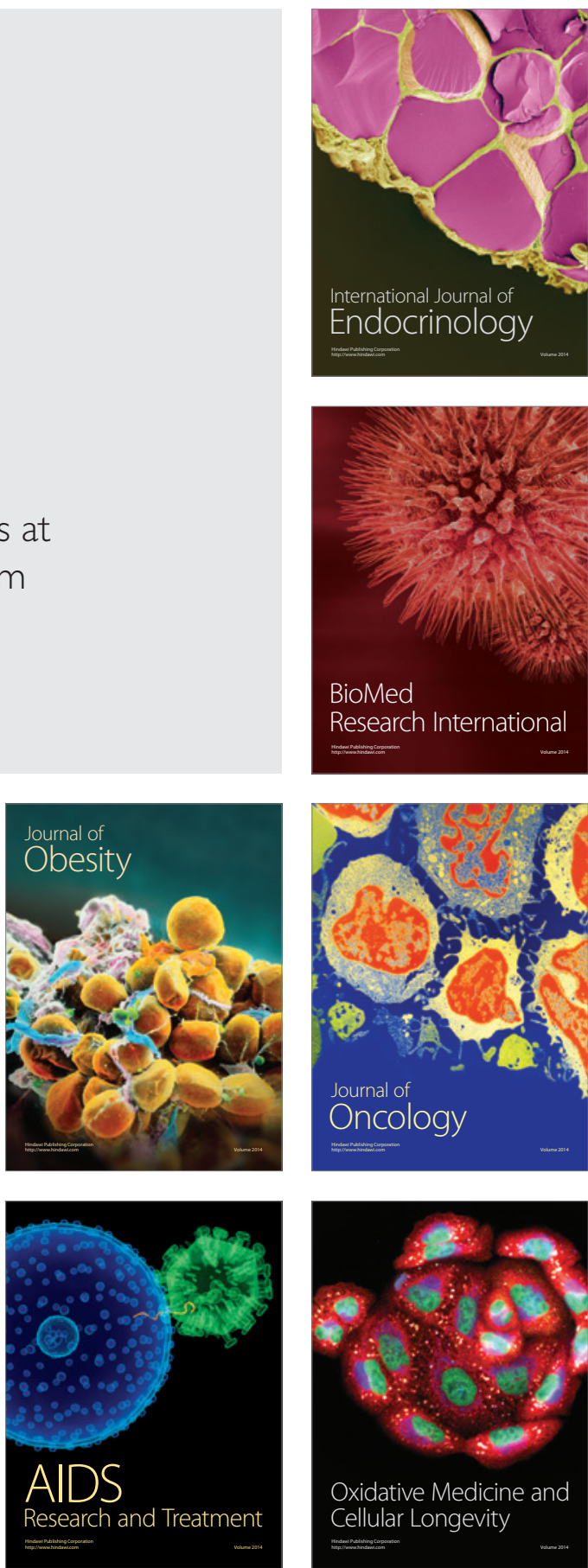